Sandra Maria Pereira de Oliveira 1

Bianca Aguiar de Morais 2

Claudia Abrantes Gonçalves 1

Cristina Maria Giordano-Dias 1

Maurício Luiz Vilela ${ }^{1}$

Reginaldo Peçanha Brazil 4

José Mario d'Almeida 3

Marise Dutra Asensi 2

Rubens Pinto Mello 1

\section{Microbiota do trato digestivo de fêmeas de Lutzomyia longipalpis (Lutz \& Neiva, 1912) (Diptera: Psychodidae) provenientes de colônia alimentadas com sangue e com sangue e sacarose}

\author{
Digestive tract microbiota in female \\ Lutzomyia longipalpis (Lutz \& Neiva, 1912) \\ (Diptera: Psychodidae) feeding on blood meal \\ and sacarose plus blood meal
}

1 Departamento de Entomologia, Instituto Oswaldo Cruz,

Fundação Oswaldo Cruz. Av. Brasil 4365 Rio de Janeiro, $R J$ 21045-900, Brasil.

2 Departamento de Bacteriologia, Instituto Oswaldo Cruz,

Fundação Oswaldo Cruz. Av. Brasil 4365,

Rio de Janeiro, $R J$ 21045-900, Brasil.

3 Departamento de Biologia Instituto Oswaldo Cruz, Fundação Oswaldo Cruz. Av. Brasil 4365,

Rio de Janeiro, $R J$ 21045-900, Brasil.

4 Centro de Pesquisa

Reneé Rachou,

Fundação Oswaldo Cruz.

Av. Augusto de Lima 1715,

C. P. 1743, Belo Horizonte, $M G$ 30190-002, Brasil.

\begin{abstract}
There are very few reports on the microbiota of the digestive tract of sand flies, an important omission considering that blood is not the only meal ingested. Male and female sand flies obtain sugar meals from several sources, thereby increasing their chance of infection with microorganisms. Chances of contamination are higher when insects are bred in the laboratory, and this may affect the development of Leishmania spp. From the digestive tract of 300 sand fly females separated in two groups we isolated 10 species of bacteria in group 1 and 8 species in group 2. In group 1, Enterobacteriaceae of the following genera were identified: Serratia, Enterobacter, and Yokenella and the non-fermenters: Pseudomonas, Acinetobacter, and Stenotrophomonas. In group 2, the Enterobacteriaceae Enterobacter and Serratia were identified as well as the non-fermenters Acinetobacter, Stenotrophomonas, Burkolderia, and Pseudomonas.
\end{abstract}

Key words Phlebotominae; Psychodidae; Microbiology

Resumo Há poucos estudos sobre a microbiota do trato digestivo de flebotomíneos, considerando-se que o sangue não é o único alimento ingerido. Os flebotomíneos, tanto os machos como as fêmeas, alimentam-se de açúcares, provenientes de várias fontes, possibilitando a ingestão de microrganismos. As chances de contaminação aumentam nos insetos criados em laboratório $e$ pode interferir no desenvolvimento da Leishmania spp. Foi separado um total de 300 fêmeas, divididas em dois lotes, das quais extraímos o trato digestivo. No lote 1(fêmeas alimentadas com sangue e sacarose) das 10 espécies bacterianas isoladas, a família Enterobacteriaceae esteve representada pelos gêneros Serratia, Enterobacter $e$ Yokenella, e o grupo dos não fermentadores pelos gêneros Pseudomonas, Acinetobacter $e$ Stenotrophomonas. No lote 2 (fêmeas alimentadas apenas com sangue) das 8 espécies isoladas o grupo dos não fermentadores esteve representado pelos gêneros Acinetobacter, Stenotrophomonas, Burkolderia e Pseudomonas, e a família Enterobacteriaceae, pelos gêneros Enterobacter $e$ Serratia.

Palavras-chave Phlebotominae; Psychodidae; Microbiologia 


\section{Introdução}

Entre os dípteros, há muitas espécies nas quais ambos os sexos alimentam-se de açúcares naturais, especialmente em néctar, seiva de plantas e secreções de afídeos. As fêmeas de algumas espécies procuram proteínas adicionais, como o sangue de vertebrados ou fluidos do corpo de outros insetos (Waterhouse, 1957).

Nos flebotomíneos, tanto os machos como as fêmeas alimentam-se de açúcares, provenientes de várias fontes, possibilitando a ingestão de microrganismos, entre eles, as bactérias presentes no trato digestivo de numerosas espécies de insetos (Tanada \& Kaya, 1993). As chances de contaminação aumentam nos insetos criados em laboratório e, geralmente, acarretam alto índice de mortalidade (Adler \& Theodor, 1927; Schlein \& Yuval, 1987; Figueiredo et al., 1997).

Segundo Adler \& Theodor (1927) e Schlein et al. (1985), a microbiota pode interferir no desenvolvimento de Leishmania spp.

Em decorrência destes fatos, desenvolvemos no presente trabalho um estudo sobre a diversidade da flora bacteriana desses insetos, em laboratório, utilizando para isso, Lutzomyia (Lutzomyia) longipalpis (Lutz \& Neiva, 1912) que é comprovadamente vetor de leishmaniose visceral nas Américas (Killick-Kendrick, 1979).

\section{Material e métodos}

Os flebotomíneos são provenientes de uma colônia de L. longipalpis originalmente capturados na Gruta da Lapinha, Município de Lagoa Santa, Minas Gerais e mantidos em laboratório no Departamento de Biologia/Instituto Oswaldo Cruz/Fundação Oswaldo Cruz, no Rio de Janeiro. Os exemplares utilizados pertenciam a 13ạ e 20a gerações. A metodologia usada na criação segue segundo Oliveira et al. (2000).

Os insetos foram separados em dois lotes de cento e cinqüenta fêmeas cada, onde o primeiro lote foi alimentado com sangue e solução de sacarose, a 50\% (w/v) e, o segundo foi alimentado somente com sangue. As fêmeas foram separadas em cinco grupos para cada alimentação, formando um pool de 30 insetos por grupo, num total de 300 fêmeas, das quais extraímos o tubo digestivo e tubos de Malpighi. No quarto dia após a emergência, as fêmeas realizaram o repasto sangüíneo, e três dias depois, foram selecionadas para dissecação. A metodologia utilizada para o isolamento e identificação das bactérias segue segundo Oliveira et al. (2000).

\section{Resultados}

Quanto aos microrganismos isolados nos dois lotes de fêmeas, predominam os bastonetes Gram negativos (BGN). As dez espécies bacterianas isoladas nas fêmeas do lote 1 , alimentadas com sangue e solução saturada de sacarose foram: Serratia marcescens, $23 \%$, Enterobacter cloacae, 17\%; do total de amostras analisadas; Pseudomonas aeruginosa, 12\%; Stenotrophomonas maltophilia, 12\%; Enterobacter aerogenes, 6,0\%; E. gergoviae, 6,0\%; E. sakazakii, 6,0\%; Yokenella regensburgei, 6,0\%; Acinetobacter lwoffii, 6,0\% e A. baumannii, 6,0\%.

Os BGN, na sua maioria, foram enquadrados no grupo dos fermentadores de açúcares, como pode-se notar com o isolamento dos gêneros: Serratia, Enterobacter e Yokenella.

O grupo dos BGN não fermentadores esteve representado pelos gêneros: Pseudomonas, Acinetobacter e Stenotrophomonas.

As oito espécies isoladas nas fêmeas do lote 2, alimentadas somente com sangue, foram: Acinetobacter baumannii, com 28,6 \% do total encontrado, seguido de Stenotrophomonas maltophilia 14\%, Burkolderia cepacia 14\%, B. gladioli $7 \%$, Pseudomonas aeruginosa 14\%, P. fluorescens 7\%, Enterobacter cloacae 7\% e Serratia marcescens $7 \%$.

Os BGN em grande parte isolados, pertenciam ao grupo dos não fermentadores de açúcares, com os gêneros: Acinetobacter, Stenotrophomonas, Burkolderia e Pseudomonas.

Os BGN fermentadores, estiveram presentes com os gêneros: Enterobacter e Serratia.

\section{Discussão}

Os dados referentes às amostras de flebotomíneos listadas permitem consignar a presença de uma microbiota bacteriana pouco diversificada no trato digestivo das fêmeas analisadas, quando comparada a outros dípteros (Szabó et al., 1966; Warburg, 1991).

No lote 1, as espécies predominantes pertencem à família Enterobacteriaceae, representada pelos gêneros Enterobacter, Serratia e Yokenella e, o predomínio dos BGN fermentadores o que confirma o observado em Diptera Ceratopogonidae, Psychodidae e Hemiptera (Jenkins, 1964; Schlein et al., 1985; Oliveira et al., 1998). Enquanto que no lote 2, os BGN, na sua maioria pertencem ao grupo dos não fermentadores de açúcares.

E. cloacae foi isolado nos dois lotes e esta espécie tem sido assinalada por diversos autores, como a espécie mais freqüente no intesti- 
no dos insetos (Rossiter et al., 1983). De acordo com Tanada \& Kaya (1993) esta é a espécie mais comumente encontrada no intestino de insetos de importância médica, inclusive em flebotomíneos provenientes do campo (Dillon et al., 1996).

Pode-se sugerir que a presença de BGN esteja associada ao modo de manutenção dos insetos no laboratório, onde eles dependem de soluções açucaradas, sangue de vertebrados, além da dieta larvar. Estas condições propiciam o desenvolvimento de bactérias do ambiente (solo, água, detritos) como os gêneros Acinetobacter, Pseudomonas, Enterobacter, Serratia e Yokenella (Koserella trabulsii), sendo alguns patógenos para as plantas como Pseudomonas e Stenotrophomonas (Holt et al., 1996).

Os gêneros Serratia, Pseudomonas, Yokenella, além de Enterobacter, já foram descritos no intestino de insetos (Moore, 1971; Holt et al., 1996; Oliveira et al.,1998).

Warburg (1991) também isolou S. marcescens em flebotomíneos adultos do gênero Phlebotomus, contudo, sabemos que $S$. marcescens e P. aeruginosa são responsáveis pela mortali- dade em colônia de L. longipalpis (Figueiredo et al., 1997), como também em outros dípteros (Pumpuni et al., 1996).

A constância das espécies bacterianas nos isolamentos nos leva a crer que façam parte de uma microbiota do inseto, em condições de laboratório. Entretanto, não se pode descartar uma possível contaminação transitória do intestino. Sabemos estar presentes as espécies $E$. cloacae, S. marcescens, A. baumannii, Stenotrophomonas maltophilia e P. aeruginosa com ou sem açúcares.

Os gêneros Pseudomonas, Acinetobacter e as espécies Stenotrophomonas maltophilia e $E$. cloacae, também foram isolados do trato digestivo de fêmeas de Lutzomyia longipalpis, provenientes do campo (Oliveira et al., 2000).

Segundo Pumpuni et al. (1996) os açúcares podem representar uma importante fonte de contaminação bacteriana. Com o presente estudo concluímos que a família Enterobacteriaceae predominou, principalmente com as espécies E. cloacae e $S$. marcescens e que desenvolveram-se melhor no trato digestivo de flebotomíneos, em presença de açúcar.

\section{Agradecimentos}

À Dra. Elizabeth Ferreira Rangel, por suas valiosas opiniões, apoio e revisão do manuscrito. À equipe do Laboratório de Transmissores de Leishmaniose e Oncocercose do Departamento de Entomologia. Ao Departamento de Biologia, por todo apoio. Ao Departamento de Bacteriologia, pela acolhida, apoio e valiosos ensinamentos. À equipe do Departamento de Medicina Tropical e na pessoa da Dra. Angela Junqueira, pelo total apoio no desenvolvimento deste trabalho.

\section{Referências}

ADLER, S. \& THEODOR, O., 1927. Attempts to transmit Leishmania tropica by bite: The transmission of L. tropica by Phebotomus sergpti. Annals of Tropical Medicine and Parasitology, 23:1-18.

DILLON, R. J.; EL KORDY, E.; LANEE, R. P., 1996. The prevalence of a microbiota in the digestive tract of Phlebotomus papatasi. Annals of Tropical Medicine and Parasitology, 90:669-673.

FIGUEIREDO, A. R.; OLIVEIRA, S. M. P.; GIORDANODIAS, C. M.; D'ALMEIDA, J. M. \& BRAZIL, R. P. 1997. Presença de Serratia marcescens e Pseudomonas aeruginosa em colônias de flebotomíneos (Diptera: Psychodidae). In: 16o Congresso Brasileiro de Entomologia, VII Encontro Nacional de Fitossanitaristas, Anais, p. 260, Salvador: 16o Congresso Brasileiro de Entomologia. 
HOLT, J. G.; KRIEG, N. R.; SNEATH, P. H. A.; STALEY, J. T. \& WILLIAMS, S. T., 1996. Bergey's Manual of Determinative Bacteriology. 9th Ed., Baltimore: Williams \& Wilkins.

JENKINS, D. W., 1964. Pathogens, parasites and predators of medically important arthropods - Annotades list and bibliography. IV. Sandflies and midges-psychodidae and ceratopogonidae. Bulletin of the World Health Organization, 30:30-32.

KILLICK-KENDRICK, R., 1979. Biology of Leishmania in phlebotomine sandflies. In: Biology of Kinetoplastida (W. H. R. Lumsden \& D. A. Evans, eds.), v. 2, pp. 395-460, London: Academic Press.

MOORE, G. F., 1971. Mortality factors caused by pathogenic bacteria and fungi of the Southern Pinee Beetle in North Carolina. Journal of Invertebrate Pathology, 17:28-37.

OLIVEIRA, S. M. P.; MORAIS, B.; GONÇALVES, C. A.; GIORDANO-DIAS, C. M.; VILELA, M. L.; BRAZIL, R. P.; D'ALMEIDA, J. M.; ASENSI; M. D. \& MELLO, R. P., 2000. Prevalência da microbiota no trato digestivo de fêmeas de Lutzomyia longipalpis (Lutz \& Neiva, 1912) (DIPTERA: PSYCHODIDAE) provenientes do campo. Revista da Sociedade Brasileira de Medicina Tropical, 33:319-322.

OLIVEIRA, S. M. P.; MORAIS, B.; GONÇALVES, C. A.; LOUREIRO, M. M.; LEANDRO, M. J. F.; GIORDANO-DIAS, C. M.; VILELA, M. L.; BRAZIL, R. P.; D'ALMEIDA, J. M.; ASENSI; M. D. \& MELLO, R. P., 1998. The microbiota of the digestive tract of females of Lutzomyia longipalpis (Diptera: Psychodidae). Memórias do Instituto Oswaldo Cruz, 93 (Sup. 2):351.
PUMPUNI, C. B.; DEMAIO, J.; KENT, M.; DAVIS, J. R. \& BEIER, J. C., 1996. Bacterial population dynamics in three anopheline species: The impact on Plasmodium sporogonic development. American Journal of Tropical Medicine and Hygiene, 54:214218.

ROSSITER, M. C.; HOWARD, D. J. \& BUCH, G. L., 1983. In Fruit Flies of Economic Importance. Rotterdam: A. A. Balkema.

SCHLEIN, Y.; POLACHECK, I. \& YUVAL, B., 1985. Mycoses, bacterial infection and antibacterial activity in sandflies (Psychodidae) and their possible role in the transmission of leishmaniasis. Parasitology, 90:57-66.

SCHLEIN, Y. \& YUVAL, B., 1987. Leishmaniasis in the Jordan Valley. IV. Attraction of Phlebotomus papatasi (Diptera: Psychodidae) to plants in the field. Journal of Medical Entomology, 24:87-90.

SZABÓ, I.; MARTON, M.; BUTI, I. \& PÁRTAI, G., 1966. Intestinal microflora of the larvae of St. Mark's fly. Acta Microbiologica Academiae Scientiarum Hungaricae, 13:47-52.

TANADA, Y. \& KAYA, H. K., 1993. Insect Pathology. New York: Academic Press.

WARBURG, A., 1991. Entomopathogens of phlebotomine sandflies: Laboratory experiments and natural infections. Journal of Invertebrate Pathology, 58:189-202.

WATERHOUSE, D. F., 1957. Digestion in insects. Annual Review of Entomology, 2:1-180. 